\title{
Molecular cloning and expression analysis of two sex-lethal homolog genes during development in the oriental river prawn, Macrobrachium nipponense
}

Y.P. Zhang ${ }^{1,2}$, H. Qiao ${ }^{3}$, W.Y. Zhang ${ }^{1}$, S.M. Sun ${ }^{3}$, S.F. Jiang ${ }^{3}$, Y.S. Gong ${ }^{3}$, Y.W. Xiong ${ }^{3}$, S.B. Jin ${ }^{3}$ and H.T. Fu ${ }^{1,3}$

${ }^{1}$ Wuxi Fishery College, Nanjing Agricultural University, Wuxi, China ${ }^{2}$ Jiangxi Fisheries Research Institute, Nanchang, China

${ }^{3}$ Key Laboratory of Freshwater Fisheries and Germplasm Resources Utilization, Freshwater Fisheries Research Center, Chinese Academy of Fishery Sciences, Ministry of Agriculture, Wuxi, China

Corresponding author: H.T. Fu

E-mail: fuht@ffrc.cn

Genet. Mol. Res. 12 (4): 4698-4711 (2013)

Received November 20, 2012

Accepted July 7, 2013

Published October 18, 2013

DOI http://dx.doi.org/10.4238/2013.October.18.8

\begin{abstract}
In this study, two $S x l$ gene homologs, designated as Mnsxll and Mnsxl2, were cloned and characterized from the freshwater prawn Macrobrachium nipponense by rapid amplification of cDNA ends. The deduced amino acid sequences of Mnsxl1 and Mnsxl2 showed high sequence homology to the insect Sxl and contained conserved domains in two RNA-binding motifs. Real-time quantitative reverse transcription-polymerase chain reaction (RT-QPCR) showed that the Mnsxll and Mnsxl2 genes were expressed in all investigated tissues, with the highest level of expression in the intestine and liver. RT-QPCR also revealed that Mnsxll and Mnsxl2 mRNAs expressions were both significantly increased at 5 and 20 days post-larvae after metamorphosis. Thus, the results of the present study imply that Mnsxll and Mnsxl2 play
\end{abstract}


complex and important roles in the sex differentiation of M. nipponense.

Key words: Macrobrachium nipponense; Sex-lethal; Sex determination; Expression pattern; Crustacean

\section{INTRODUCTION}

Sex-determining cascades in insects constitute an intensively studied system that evolves from bottom to top (Wilkins, 1995). As an RNA-binding protein, the gene Sxl controls sex determination, dosage compensation, and oogenesis in Drosophila melanogaster (Penalva and Sánchez, 2003; Serna et al., 2004). Its state of activity is set at around the blastoderm stage in somatic cells by the primary sex-determining signal, which is formed by the ratio of $\mathrm{X}$ chromosomes to sets of autosomes. If this ratio is 1.0 (XX:AA), $S x l$ is activated and a female develops. Alternatively, a ratio of 0.5 (X:AA) leaves $S x l$ inactive, and male development ensues (Cline and Meyer, 1996; Schütt and Nöthiger, 2000). So far, the fundamentals of the molecular regulation mechanism of the sex-determination pathway of Drosophila have been elucidated (Casper and Van Doren, 2009; Siwicki and Kravitz, 2009), but knowledge regarding sex determination in other insects remains at the chromosome or gene level.

Understanding tissue- and stage-specific gene regulation remains one of the central issues in developmental biology. $S x l$ was initially discovered as a master switch gene of sex determination in Drosophila (Cline, 1978). The Sxl gene has been implicated in germ cell proliferation, differentiation, and meiotic recombination during early oogenesis in Drosophila (Vied and Horabin, 2001; Vied et al., 2003). Sxl has also been isolated and characterized in other species such as Chrysomya rufifacies (Müller-Holtkamp, 1995), Megaselia scalaris (Sievert et al., 2000), Ceratitis capitata (Saccone et al., 1998), Sciara ocellaris (Ruiz et al., 2003) and Bactrocera oleae (Lagos et al., 2005). Although the $S x l$ sequence from these species shares a high degree of similarity with that from Drosophila, it is not sex-specifically spliced and does not appear to play the key discriminative role in controlling sex determination and dosage compensation that it plays in Drosophila (Traut et al., 2006). Considering the fact that crustaceans have a dramatically close evolution relationship with insects (Glenner et al., 2006; Budd and Telford, 2009), we hypothesized that the homolog Mnsxl from the oriental river prawn Macrobrachium nipponense is involved in the regulation of sex determination in crustaceans.

M. nipponense (Crustacea; Decapoda; Palaemonidae) is a commercial freshwater prawn. It is considered as an important fishery resource in China, with an annual production of 205,010 tons (Bureau of Fishery, Ministry of Agriculture, P.R.C., 2009). Male individuals of the oriental river prawn grow faster and reach higher weights at harvest than female individuals reach. Thus, culture of all-male populations would be dramatically economically beneficial. To understand the mechanism controlling sex maturation, a high-quality normalized cDNA library from testis tissue of this species has been established (Qiao et al., 2012). For this reason, molecular mechanisms regulating the expression of sex-specific and sex-determining genes in crustaceans have received great attention in recent years (Li et al., 2009; Kato et al., 2011; Ventura et al., 2012). In the present study, we aimed to obtain a full cDNA clone encoding the $S x l$ gene from the cDNA library of testis in M. nipponense, and to examine the expression pattern of the $S x l$ gene during the embryonic development, larvae, and post-larvae 
stages of M. nipponense. This study may provide insight into the regulatory mechanism of sex determination in the oriental river prawn.

\section{MATERIAL AND METHODS}

\section{Tissue preparation}

Several healthy adult oriental river prawns with wet weight of 1.26 to $4.25 \mathrm{~g}$ were obtained from Tai Lake in Wuxi, China $\left(31^{\circ} 28^{\prime} 22^{\prime \prime} \mathrm{N}, 120^{\circ} 13^{\prime} 44^{\prime \prime} \mathrm{E}\right)$. All of these samples were transferred to laboratory breeding conditions and maintained in a 500-L tank with aerated freshwater for $72 \mathrm{~h}$ before tissue collection. The different developmental stages of eggs and larvae were obtained from our breeding room. After prawn spawning, each developmental stage of embryos was collected as per the morphological methods, following the criteria of Chen et al. (2012). Larvae were collected every 4 days between 1 day post-hatching (L1) and L13 (1 day before the metamorphosis). Post-larvae were collected every 5 days between 1 and 20 days after the metamorphosis (P1 P20), and every 10 days between P20 and P30. Several tissues, including ovary, testis, muscle, heart, abdominal ganglion, brain, liver, and intestine, were also collected. The samples were washed with $1 \mathrm{X} 0.01 \mathrm{M}$ phosphate-buffered saline, frozen directly in liquid nitrogen, and stored at $-80^{\circ} \mathrm{C}$ until processed.

\section{Total RNA extraction and reverse transcription}

Total RNA was extracted using RNAiso Plus Reagent (TaKaRa, Japan) in accordance with the manufacturer protocol. The isolated RNA was treated with RNase-free DNase I (Sangon, Shanghai, China) to eliminate possible genomic DNA contamination. The concentration of each total RNA sample was then measured using a BioPhotometer (Eppendorf), and $2 \mu \mathrm{L}$ was analyzed on a $1 \%$ agarose gel to check the integrity. The cDNA was synthesized from $5 \mu \mathrm{g}$ total RNA using the PrimeScript ${ }^{\mathrm{TM}}$ RT-PCR Kit (TaKaRa) according to manufacturer protocols. The cDNA was kept at $-20^{\circ} \mathrm{C}$ for real-time quantitative reverse transcription-polymerase chain reaction (RT-QPCR).

\section{Rapid amplification of cDNA ends}

Four gene-specific primer sets (Table 1) were designed on the basis of the expressed sequence tag of the sex-lethal homolog (GenBank accession No. JK526786) and sex-lethallike protein (GenBank accession No. JK525705) obtained from the M. nipponense testis cDNA library. The full lengths of the Mnsxl1 and Mnsxl2 genes were obtained by using the SMART $^{\mathrm{TM}}$ RACE cDNA Amplification Kit (Clontech, USA). For 5'-rapid amplification of cDNA ends (RACE), the primer sets consisted of 5'-CDS primer A (as the RT primer); four gene-specific primers of GSP1, GSP2, GSP3, and GSP4; and universal primer A mix (UPM) (Table 1). For 3'-RACE, the primer sets consisted of 3'-CDS primer A (as the RT primer); four gene-specific primers of GSP5, GSP6, GSP7, and GSP8; and UPM (Table 1). The same PCR conditions were followed as described in the manufacturer protocol of the Advantage ${ }^{\mathrm{TM}} 2$ PAC Kit (Clontech). 


\begin{tabular}{|c|c|c|}
\hline Primer & Nucleotide sequence $\left(5^{\prime} \rightarrow 3^{\prime}\right)$ & Code \\
\hline \multicolumn{3}{|l|}{ Primers for $5^{\prime}$ RACE PCR } \\
\hline MnSx11 5' GSP primer 1 & GCCAGGAGAAGACATAAAG & GSP1 \\
\hline MnSxl1 5' GSP primer 2 & GGAGTCGGGTTTGTCAGGT & GSP2 \\
\hline MnSxl2 5' GSP primer 1 & GCGTGGGAACTACAACAA & GSP3 \\
\hline MnSxl2 5' GSP primer 2 & CAGCAGGGTGGTAAGATG & GSP4 \\
\hline \multicolumn{3}{|l|}{ Primers for 3' RACE PCR } \\
\hline MnSxl1 3' GSP primer 1 & CCACCTGTGATGATACTTGACCTCC & GSP5 \\
\hline MnSx11 3' GSP primer 2 & TCTAACTGGTCTAGGGTGTAGGATCTGG & GSP6 \\
\hline MnSx12 3' GSP primer 1 & GTAACCACCACCAGTGCCAATGG & GSP7 \\
\hline MnSxl2 3' GSP primer 2 & TCGGCTCAGTACCACCTGTGATG & GSP8 \\
\hline \multicolumn{3}{|l|}{ Clontech $^{\mathrm{TM}}$ Kit primesr } \\
\hline Universal primer A mix & CTAATACGACTCACTATAGGGCAAGCAGTGGTATCAACGCAGA GT & \\
\hline \multicolumn{3}{|c|}{ Primers for real-time PCR analysis } \\
\hline MnSxl1 5' primer & AAGGTGGCACGGAACCGTTGG & RT-F1 \\
\hline MnSxl1 3' primer & CCACCTGTGATGATACTTGACCTCC & RT-R1 \\
\hline MnSxl2 5' primer & GCGTGGGAACTACAACAA & RT-F2 \\
\hline MnSxl2 3' primer & TCGGCTCAGTACCACCTGTGATG & RT-R2 \\
\hline$\beta$-actin 5' primer & TATGCACTTCCTCATGCCATC & $\beta$-actinF \\
\hline$\beta$-actin $3^{\prime}$ primer & AGGAGGCGGCAGTGGTCAT & $\beta$-actinR \\
\hline
\end{tabular}

\section{Primer sequences}

The PCR products were gel-purified and ligated into the pMD18-T vector (TaKaRa) following the instructions provided by the manufacturer. The recombination was then transformed into Escherichia coli DH5 $\alpha$ (Qiagen) competent cells, which were identified by blue/ white screening and confirmed by PCR. Three of the positive clones were sequenced in both directions using an automatic DNA sequencer (ABI Applied Biosystems Model 3730) and these resulting sequences were verified and subjected to cluster analysis by using the online database of the National Center for Biotechnology Information (NCBI).

\section{Nucleotide sequence and bioinformatic analyses}

The searches for protein sequence similarities were conducted with the BLAST algorithm at the NCBI (http://www.ncbi.nlm.nih.gov/BLAST/). The protein prediction was performed using the ORF finder (http://www.ncbi.nlm.nih.gov/gorf/). The ProtParam program (http://www.expasy.ch/tools/protparam. html) was used to compute physical and chemical parameters of the amino acid sequence. The motif was searched with the motif scan program (http://hits.isb-sib.ch/cgi-bin/motifscan/). Sxl deduced amino acid sequences from M. nipponense and representative invertebrates were compared by multiple sequence alignment using ClustalX and the Box shade program (http://www.ch.embnet.org/software/BOX_form.html). A neighbor-joining phylogenetic tree was constructed using the MEGA version 4 software.

The Mnsxll and Mnsxl2 mRNA expressions at different stages, from embryo to post-larval, and in various adult tissues were measured by a SYBR Green real-time quantitative RT-PCR analysis in the CFX96TM Real-Time System (Bio-Rad, USA). Gene-specific primers (Table 1) were used to amplify the Sxl transcript, and the PCR products were sequenced to verify the specificity of the PCR primers. The $\beta$-actin primers (Table 1 ) were used to amplify the $\beta$-actin fragments that were used as an internal control. Amplifications were performed in a 96-well plate with a $20 \mu \mathrm{L}$ reaction volume containing $10 \mu \mathrm{L} 2$ SYBR Green Premix Ex Taq (TaKaRa), $0.4 \mu \mathrm{L}$ 
each $2 \mu \mathrm{M}$ primer, $2 \mu \mathrm{L}$ template, and 7.2 $\mu \mathrm{L}$ PCR-grade water. The thermal profile for SYBR Green real-time quantitative RT-PCR was $95^{\circ} \mathrm{C}$ for $10 \mathrm{~min}$, followed by 40 cycles of $95^{\circ} \mathrm{C}$ for $15 \mathrm{~s}$ and $60^{\circ} \mathrm{C}$ for $1 \mathrm{~min}$. DEPC-water was used as a negative control instead of the template. A relative standard curve was constructed using 10-fold serially diluted cDNA. Each sample was run in triplicate along with the internal control gene. To ensure that only one PCR product was amplified and detected, a dissociation curve analysis of amplification products was performed at the end of each PCR. The relative copy number of Mnsxll and Mnsxl2 mRNA was calculated according to the $2^{-\triangle \Delta C T}$ comparative CT method (Livak and Schmittgen, 2001).

\section{Statistical analysis}

All data are reported as means $\pm \mathrm{SE}$ (standard error; $\mathrm{N}=3$ ). Statistical analysis was performed using the SPSS 13.0 software. Statistical significance was determined using one-way ANOVA and post hoc Duncan multiple range tests. Significance was set at $\mathrm{P}<0.05$.

\section{RESULTS}

\section{Cloning and identification of the Mnsxl cDNA}

The full-length Mnsxl1 cDNA was 1138 bp, and it included a 927-bp open reading frame (ORF) encoding a 308-amino acid protein with an estimated molecular mass of 33.404 $\mathrm{kDa}$ and isoelectric point of 8.71. The 5'- and 3'-untranslated regions (UTRs) were $104 \mathrm{bp}$ and $107 \mathrm{bp}$, respectively (Figure 1). Mnsxl2 cDNA, which was 1214 bp long, contained a 726-bp ORF and encoded a 241-amino acid protein with a predicted molecular mass of $26.706 \mathrm{kDa}$ and an isoelectric point of 9.31. The 5'- and 3'-UTRs were $103 \mathrm{bp}$ and $385 \mathrm{bp}$, respectively (Figure 2). The Mnsxl1 and Mnsxl2 cDNA sequences have been submitted to GenBank under the accession Nos. JX457334 and JX457335, respectively. The conserved sequence and characteristic motifs of two RNA recognition motifs domains were identified in the deduced amino acid sequences of Mnsxl1 and Mnsxl2, and the conserved motifs were located in the $\mathrm{N}$-terminal region and central region, respectively (Figure 3).

\section{Homology and phylogenetic analysis of Mnsxl}

The amino acid sequences of Mnsxl1 and Mnsxl2 were compared with the sequences of previously reported Sxl proteins, showing that the Mnsxll protein shared high identity to those of other species such as Harpegnathos saltator (EFN79874.1; 66\%), Daphnia pulex (EFX75394.1; 62\%), Bombyx mori (BAE86938.1; 59\%), and Rhynchosciara americana (AAS45604.1; 56\%). Mnsxl2 shared 67\% identity to the sex-lethal-like protein of Acromyrmex echinatior (EGI69813.1), 64 and 63\% identity to the sex-lethal protein variant 2 (EFX75395.1) and variant 1 (EFX75394.1) of D. pulex, respectively, and 51\% identity to the Sxl homolog of Lepeophtheirus salmonis (ACO12409.1). A neighbor-joining phylogenic tree was constructed based on reported arthropod Sxl amino acid sequences by using the MEGA 4.0 software. The tree constructed by the neighbor-joining method showed that Mnsxl1 and Mnsxl2 were not lined with crustaceans but with the Hymenoptera (Figure 4), suggesting a closer phylogenetic relationship with the latter. 


\begin{tabular}{|c|c|}
\hline & \\
\hline & \\
\hline 1 & CTACTGGGTCAGCACTCCCTGATGGGGAGACAAGAACAAATTTAATCATCAATTACCTTC \\
\hline & 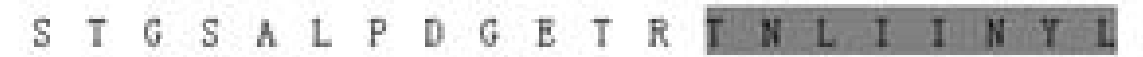 \\
\hline 1 & CACAGAOGCTAACTGAOCAAGAGTIIIATAAAATIITGTGTGGTGGGCOCTATCAAA \\
\hline & $\begin{array}{llllllllllllllllllll} & Q & T & L & T & D & Q & E & F & Y & K & I & F & V & V & V & G & P & I & B\end{array}$ \\
\hline 1 & ATTGCOGGATCATGAAAGATTIAAGCAGACTGGGTATTCGTTTGCGПTСССТTCGTGG \\
\hline & 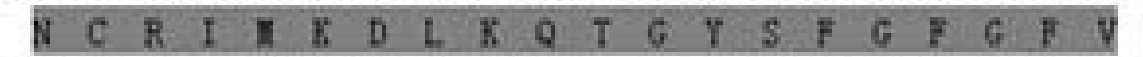 \\
\hline 1 & AGTATCAGAAMOCAGAAGATGCTCCTAMGGCAATTCTTCAGTTAAATAATCTTCCTGTGC \\
\hline & $\begin{array}{llllllllllllllllllll} & F & Q & K & P & E & D & A & A & K & A & I & L & Q & L & N & N & L & P & V\end{array}$ \\
\hline & AACATAAACGTATCAAGGTITCTTACGCTOGGCOGCCAGGAGAAGACATAAAGGAGACAA \\
\hline & 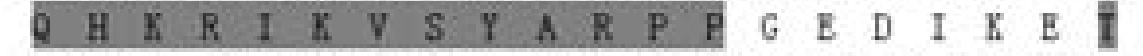 \\
\hline & 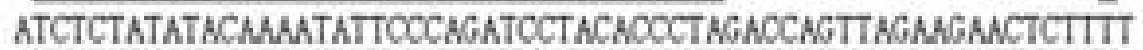 \\
\hline & 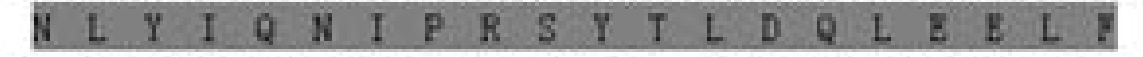 \\
\hline & 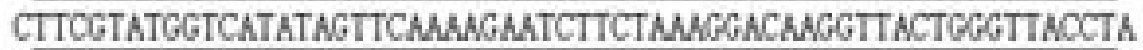 \\
\hline & $\begin{array}{llllllllllllllllllll} & S & Y & G & H & I & V & 0 & \text { K } & \text { N } & \text { L } & \text { L } & \text { K } & \text { D } & \text { K } & \text { V } & \text { T } & G & \text { L } & \text { P }\end{array}$ \\
\hline & GAGGAGTCGGGIITGTCAGGIITGATARGAAGAGOGAGGCAGAAGCTGCTATCAGTGGCA \\
\hline & 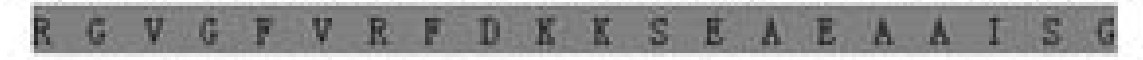 \\
\hline & TGAATCGTGTTACTCOGGAAGGTCGCACCGAACCGTTCGTAGTCAGAGTACCAGAAGACC \\
\hline & 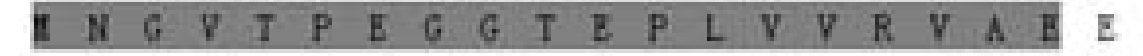 \\
\hline & ATGGAAAAATGAAMGCAGCTTACTATGCTGGGTATCATGCAGGACTAAACAACACGAGAG \\
\hline & 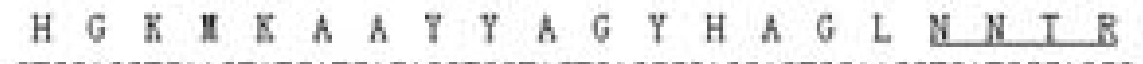 \\
\hline & GTGGAGGTCAAGTATCATCACAGGTGGTACTGAGOCGAGGAGTGGAMGGTGTGCCAGCG \\
\hline & 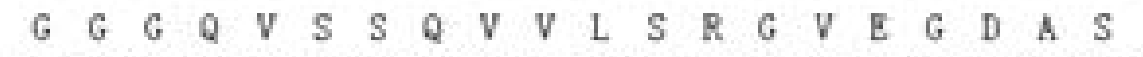 \\
\hline & 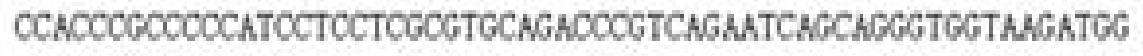 \\
\hline & A $T$ T R P H \\
\hline & CCOCAGACAGGGTCGGTANCOGCTATANCCOCATTGGCACTGGTGGTGGTTACGGTGGTG \\
\hline & 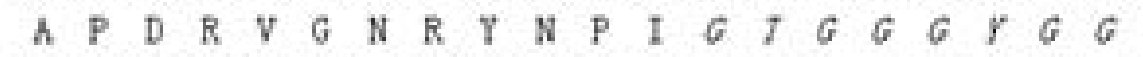 \\
\hline & GTATGGGTGGAGGTTATGGOGGTGTGCGTAGCTCTGCAGAATCATTCTAOCAGTTCTCCA \\
\hline & $\begin{array}{llllllllllllllllllll} & \boldsymbol{X} & G & G & G & Y & G & G & V & G & \mathrm{~S} & \mathrm{~S} & \mathrm{~A} & \mathrm{E} & \mathrm{S} & \mathrm{F} & \mathrm{Y} & Q & \mathrm{~F} & \mathrm{~S}\end{array}$ \\
\hline & CADOGADGTTCAGTCGGGATGACTACAOCTCATTCTCAAACATCGATCCTGGAGGCTACG \\
\hline & $\begin{array}{llllllllllllllllllll} & P & T & F & S & G & D & D & Y & T & S & F & S & N & N & D & A & G & G & Y\end{array}$ \\
\hline & tgcacagcatat ccatgttt tgagtgtcattgctaggttce \\
\hline & $R Y *$ \\
\hline & t tgattttgtagaagasasasasoas \\
\hline
\end{tabular}

Figure 1. Mnsxl1 nucleotide (above) and deduced amino acid (below) sequences. 


\begin{tabular}{|c|c|}
\hline & tetetst \\
\hline$\$ 1$ & GTTITGTTTATTTAGGTTAGOOGTGAGGTTTATAMAGCATCATGAGTITCAGTCATC \\
\hline & $1 \mathrm{SE} \mathrm{ES}$ \\
\hline 21 & 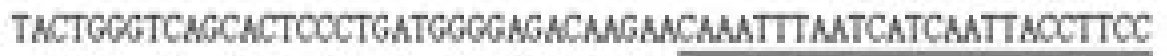 \\
\hline & 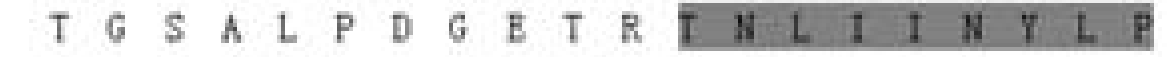 \\
\hline $\mathbb{1}$ & MCNGACOCTAMTGMOCANGGTITATAMATTITGTTGTCGTOGGCOCTATCMAMA \\
\hline 7 & Q T L L T D Q E E F \\
\hline 41. & 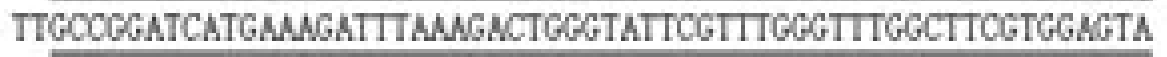 \\
\hline$\pi$ & E R I II K D L K T G F S F G F G F V E F \\
\hline 01 & TCAGAACCAGAGATGCTGCTAAGOCAMTICTCAGTTAATAACTICCTGTGCAMA \\
\hline 7 & 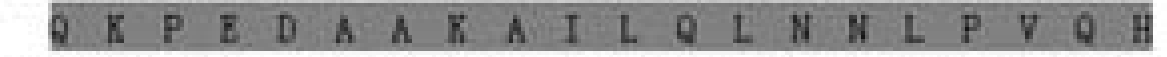 \\
\hline 61 & TAACGIATCAAGIIICTIAOCCTOGGCOCCCAGGAGAAGACATAAGGAGACAATCT \\
\hline 7 & 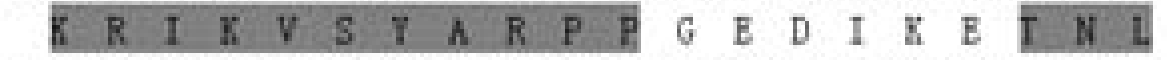 \\
\hline 121 & CTATATACAMATATIOCCAGATOCTACACOCTAGACCAGITAGAGGACTCTIICTIC \\
\hline 07 & 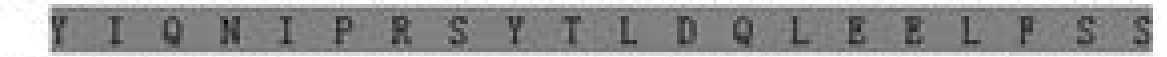 \\
\hline 481 & GTATGGTCATATAGTICAAAGAATCTICTAAOGACAAGGTIATGGGTLCCTAGAGG \\
\hline 27 & 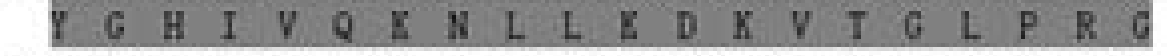 \\
\hline 541 & 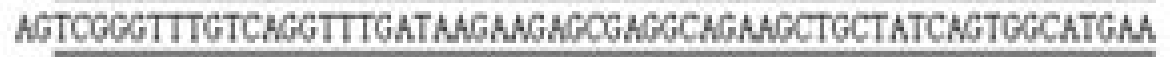 \\
\hline 147 & 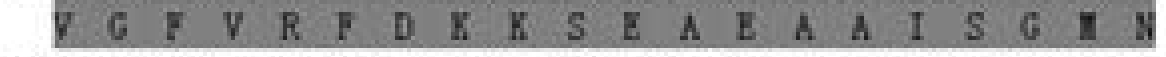 \\
\hline 501. & TOCTGTTACTOOGGAMGGTCCCAOGOAOCOTTOGTAGTCAMAGTACCAGMGMACATGG \\
\hline 67 & E V I P E G G J E P L V V V K V A E E H G \\
\hline 361 & MAMATCMAOCAOCTTMCTATCCTOCOTRTCATOCAOONCTAMCACACGMONGGTGG \\
\hline 87 & 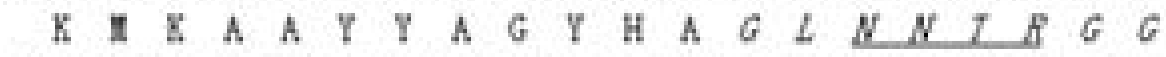 \\
\hline 721 & 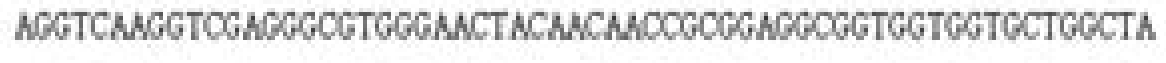 \\
\hline 07 & 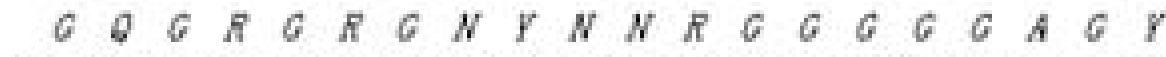 \\
\hline 81 & CCAMGGACGTGGAACTATAACAATGTGTATCATCACAGGTGGTACTGAGCCgaBSagts \\
\hline 127 & 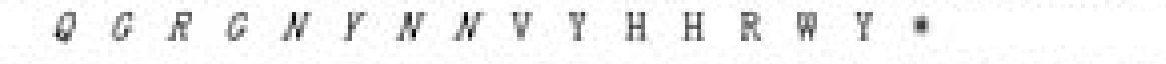 \\
\hline 81 & ccassgacgt grasactatascastigtgtatcatcacagst ggtactgagccgageatg \\
\hline 841 & 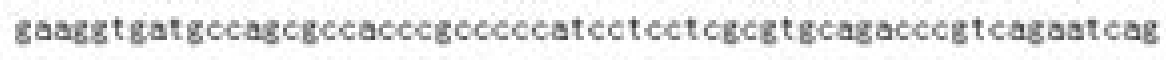 \\
\hline 901 & cagestegtagategcccogacagestcegtaaccetataacceat tescactegt \\
\hline 961 & 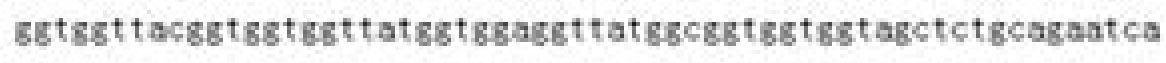 \\
\hline 1021 & ttctaccagt tetccacacegact teagt tgegatgactacacctcattctcaacatg \\
\hline 081 & gatgctgeagetacgecggtattagtctggeatgeacagcatatecatgtt tgagt \\
\hline 141 & 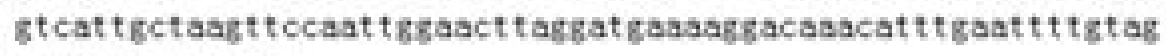 \\
\hline 201 & \\
\hline
\end{tabular}

Figure 2. Mnsxl2 nucleotide (above) and deduced amino acid (below) sequences. 

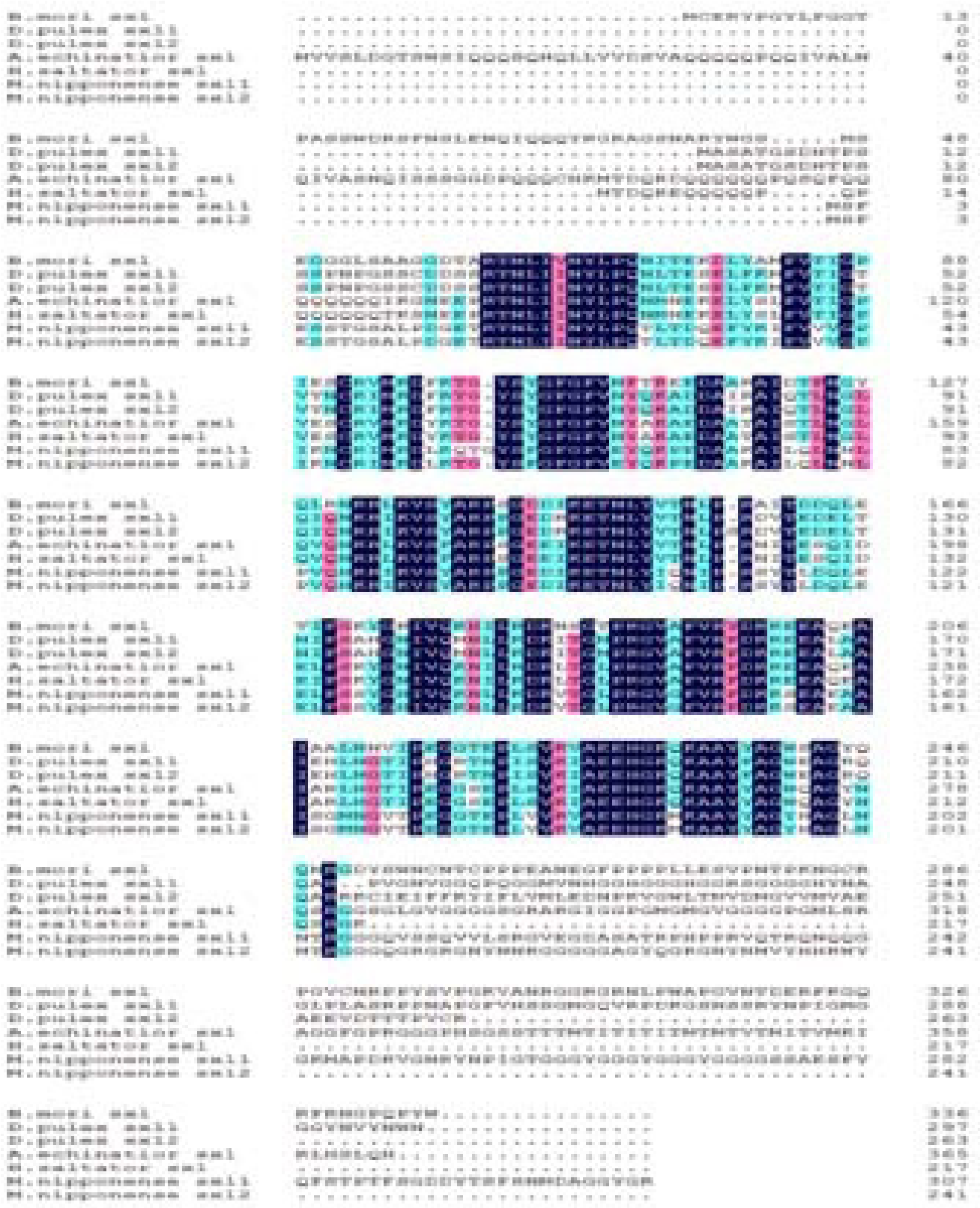

Figure 3. Alignment of Macrobrachium nipponense Sxl amino acid sequences with other species using DNAMAN. 


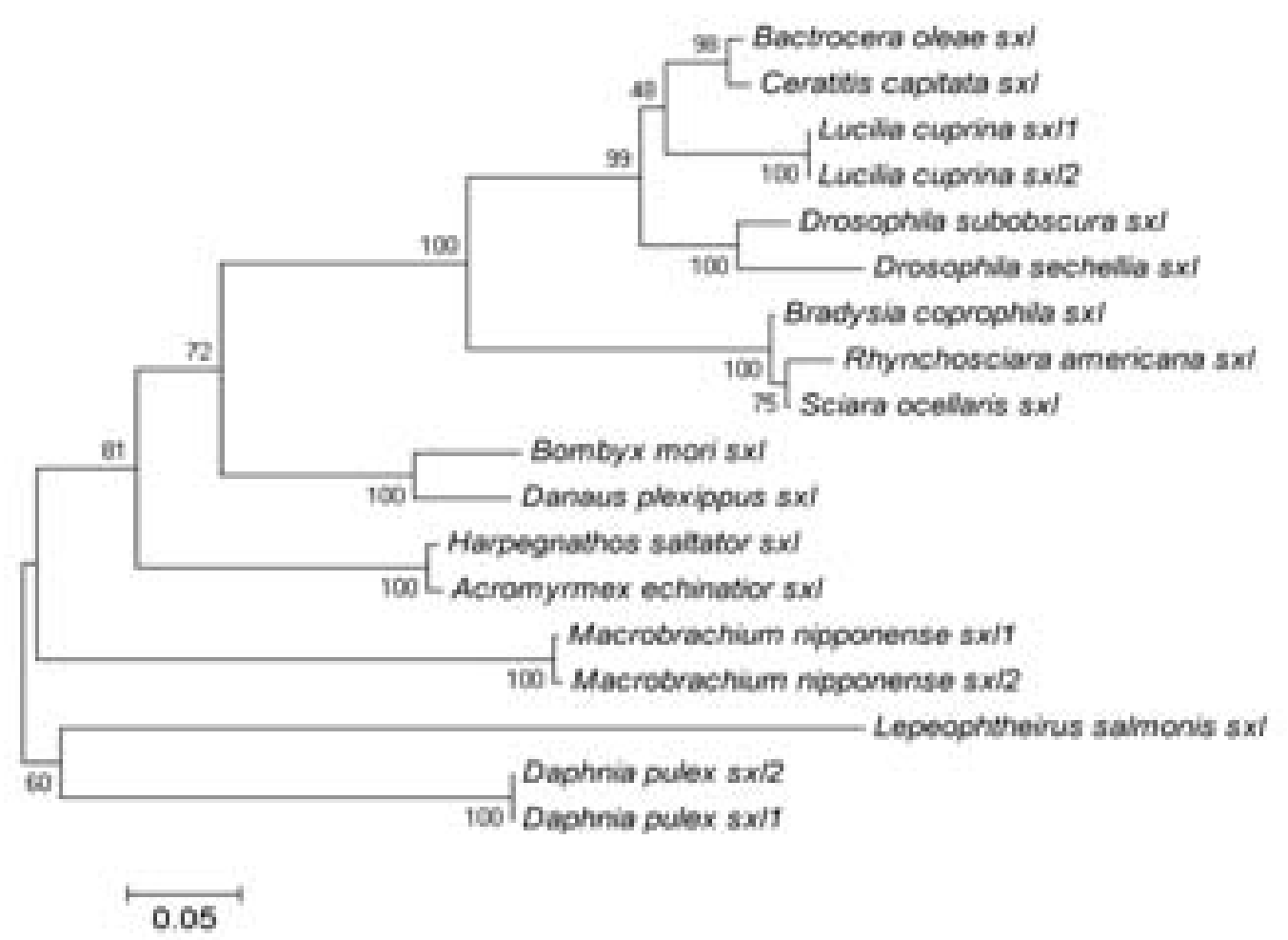

Figure 4. The neighbor-joining phylogenetic tree based on the sequences of Sxl from different species.

\section{Tissue distribution of Mnsxl1 and Mnsxl2 mRNA}

Real-time quantitative RT-PCR analysis of Mnsxl mRNA indicated that the highest expression level was in the intestine (54.19-fold), followed by the liver (43.7-fold), muscles (9.06-fold), and testis (7.32-fold). The lowest levels were detected in the ovary tissue (1-fold). Similarly, the highest level of Mnsxl2 was observed in the liver (52.18-fold) and then intestine (17.8-fold), and the lowest level was detected in the abdominal ganglion (1-fold) followed by that in muscles (1.23-fold) (Figure 5).

\section{Expression analysis of Mnsxl1 and Mnsxl2 mRNA during embryo, larvae, and post-larvae stages}

Mnsxl mRNA expression levels were studied by performing real-time quantitative RT-PCR on embryos at different developmental stages, the larval and post-larval stages. The results revealed that both Mnsxl genes were expressed in all the developmental stages of the M. nipponense embryo. The expression level of Mnsxll increased gradually from the cleavage stage (CS) to the gastrula stage (GS), and it was the highest at GS. Subsequently, it maintained a steady level from GS to the protozoea stage (PS), but declined abruptly at the zoea stage (ZS). However, the expression of Mnsxl2 was slightly stronger in CS than in the blastula stage 
(BS). After decreasing to a low level, the expression of Mnsxl2 gradually increased with the development of the embryo and peaked at the nauplius stage (NS), where it maintained a steady level up to PS, and then again abruptly decreased at ZS (Figure 6).
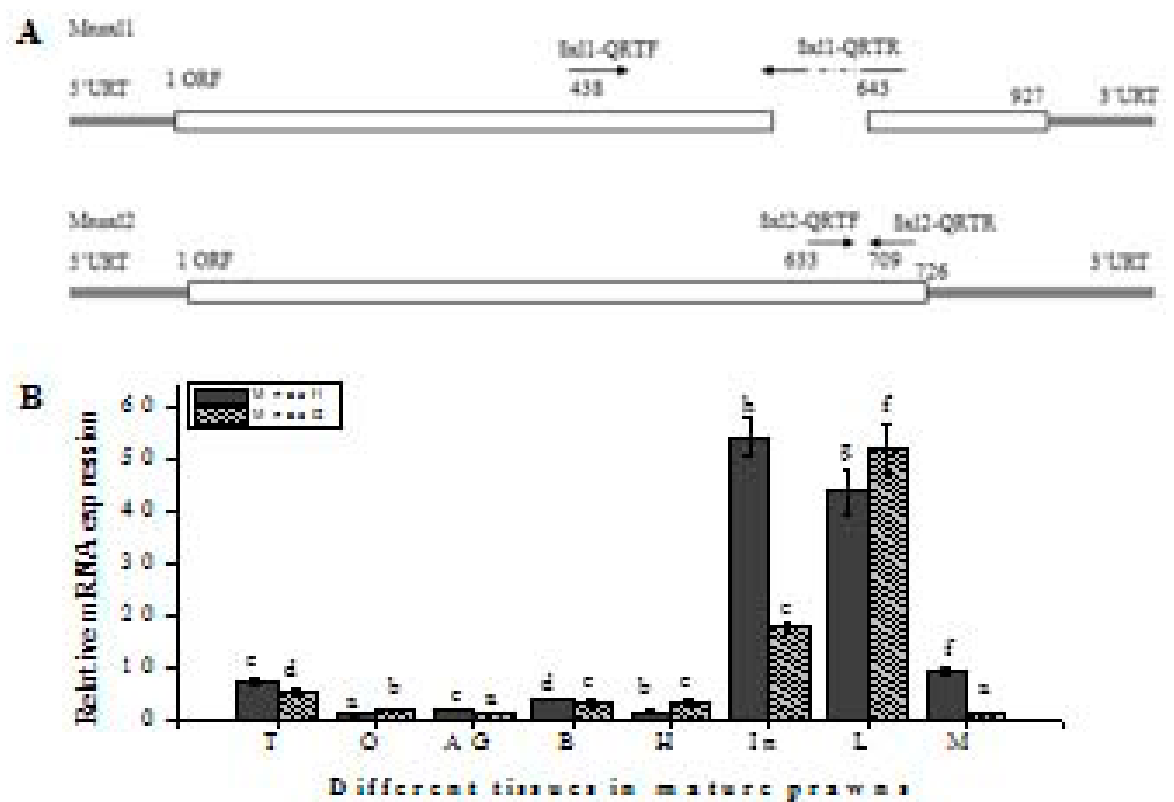

Figure 5. A. Diagrammatic representation of primer locations used in real-time quantitative RT-PCR. B. Relative expression levels of Mnsxll and Mnsxl2 transcripts quantified in various adult tissues.

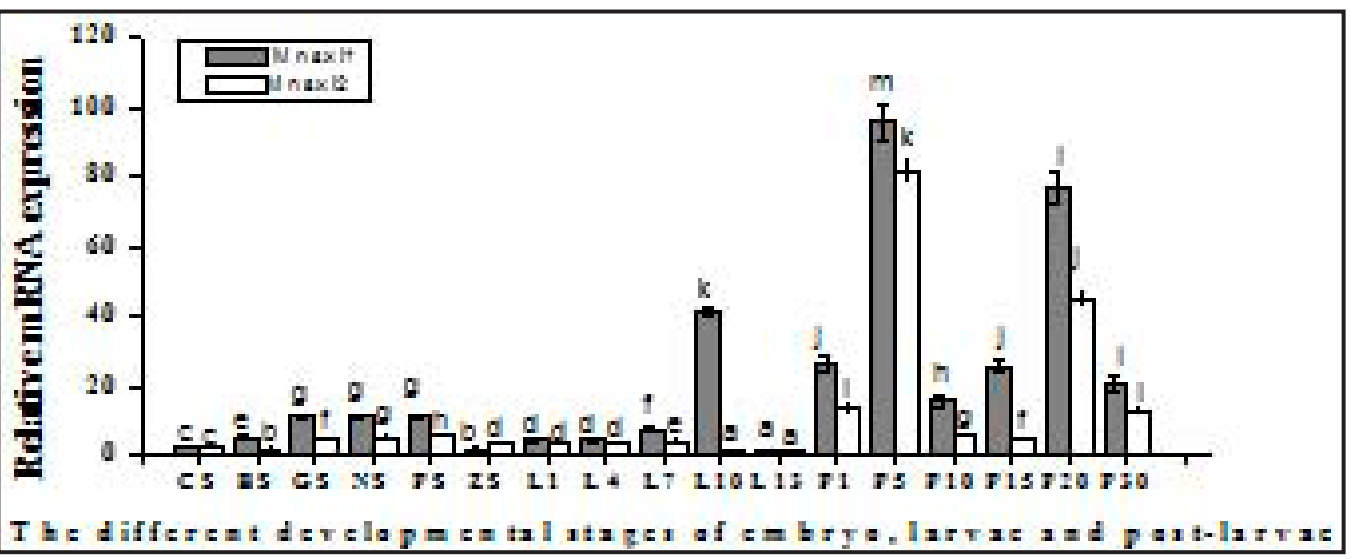

Figure 6. Mnsxl1 and Mnsxl2 mRNA expressions, normalized to $\beta$-actin, quantified in Macrobrachium nipponense during the embryos, larvae before the metamorphosis, and post-larvae after the metamorphosis.

During the larvae stage, the expression levels of Mnsxl1 and Mnsxl2 increased gradually with each day of larvae and reached the maximum at L10 (41.26-fold) or L7 (3.71-fold), respectively. The lowest expression levels of the Mnsxls were found at L13, as well as 1 
day before metamorphosis (Figure 6). After metamorphosis, the larvae transitioned into postlarvae that resemble miniature adults. During the post-larval stage, the expression levels of Mnsxl1 and Mnsxl2 gradually increased from P1 to P5, reaching the highest level at P5 (96.18fold and 81.66-fold. respectively), and then gradually decreased from P10 to P15. Subsequently, the Mnsxl1 and Mnsxl2 levels abruptly increased again at P20 (79.65-fold and 45.11-fold, respectively), but also abruptly declined at P30 (Figure 6).

\section{DISCUSSION}

The sex-lethal gene $(S x l)$ is a master switch gene for sex determination. The sexlethal protein belongs to the family of RNA-binding proteins, acting as a regulator of both alternative pre-mRNA splicing and translation (Sievert et al., 2000). In the present study, we cloned and identified two $S x l$ homologs in M. nipponense that we named Mnsxl1 and Mnsxl2. The nucleotide sequence analysis showed that Mnsxll and Mnsxl2 shared 92.75\% identity, but the Mnsxl1 cDNA sequence lacked 80 bp compared with the sequence of Mnsxl2. Thus, we speculated that they are isoforms, which are rather common with Sxl and its homologs in insectssuch as Lucilia cuprina and D. pulex (Traut et al., 2006). The Sxl homologs of fly species contained three highly conserved domains, including two RNA-binding domains (RBD) and an Sxl-specific domain at the N-terminus (Sievert et al., 2000). The conserved sequence and characteristic motifs of RNA recognition motifs were identified in the deduced amino acid sequences of Mnsxll and Mnsxl2, which endow the Sxl protein with the capacity to bind to RNA. The Sxl-specific domain in the Sxll and Sxl2 nucleotide sequences of M. nipponense was not observed, a reasonable explanation being that the Sxl-specific domain is not characteristic for Sxl in a wider range of insects; it was the most well-conserved part of this region in Diptera, but was not recognizable in non-dipterans (Traut et al., 2006). Compared with other invertebrate nucleotide sequences (Sievert et al., 2000; Lagos et al., 2005), the highest degree of similarity was observed in their two RBD domains (97.60 100\%), suggesting that Mnsx11 and Mnsxl2 were likely to perform similar functions for the capacity to bind to RNA. The Cterminal of the two isoforms was significantly different, suggesting that they have different spatial conformations and functions. Phylogenetic analysis revealed that Mnsxl1 and Mnsxl2 were closely related to Hymenoptera Sxl, but separated from their homologs of crustacean $L$. salmonis and $D$. pulex. The exact orthologous relation for Mnsxls needs further study when more Sxls are cloned from crustaceans.

Apart from Drosophila, Sxl has also been transcribed in adult gonads and somatic tissues of both sexes in S. ocellaris and B. oleae by northern blot analyses (Maine et al., 1985; Ruiz et al., 2003; Lagos et al., 2005), and $S x l$ expression was found to be extraordinarily high in the ovaries (Sievert et al., 2000; Ruiz et al., 2003; Cline et al., 2010). The current study was the first to report Mnsxl gene expression in the abdominal ganglion, which is part of the central nervous system in shrimp. In the examined tissues, the abdominal ganglion is the only organ reported to secrete hormones to induce ovary maturation in crustaceans (Kulkarni et al., 1991; Jin et al., 2003). Interestingly, the highest levels of Mnsxll and Mnsxl2 mRNAs were detected in intestine and liver tissue, respectively, suggesting that Mnsxls are involved in other physiological functions of the shrimp.

The developmental process of oocytes in the prawn undergoes a series of cellular differentiations, in which differential genes are expressed temporally and spatially to en- 
sure the proper development of oocytes (Qiu et al., 2005; Meeratana and Sobhon, 2007). In the present study, we found that the Mnsxll and Mnsxl2 mRNAs were expressed at every stage, including the cleavage stage during the embryonic development of M. nipponense, and had a similar expression pattern except at the blastula stage. It is believed that high levels of Sxl protein in fertilized eggs were derived from zygotic expression of the Sxl gene, and maternal Sxl mRNAs were not translated prior to fertilization (Salz et al., 1989; Bopp et al., 1991). The results of the present study showed that the expression of Mnsxll was gradually increased with the development of the embryo, and reached a peak at the nauplius stage. The level of Mnsxl2, however, decreased at BS, and then gradually increased and peaked at PS, which was similar to the finding reported in the previous study (Bell et al., 1988; Keyes et al., 1992) on Drosophila that Sxl mRNA expression is zygotically activated at the blastoderm stage and decreased at late embryonic development. Because embryonic organogenesis of $M$. nipponense starts from the nauplius stage and is most active at the protozoea stage (Zhang et al., 2010), the expressions of Mnsxl1 and Mnsxl2 during embryogenesis and organogenesis in this study imply that Mnsxl1 and Mnsxl2 may play different roles in the embryonic development of $M$. nipponense.

During the larvae stage, Mnsxll and Mnsxl2 mRNA expressions gradually increased with the age of larvae and respectively peaked at L10 and L7 before the metamorphosis, but decreased to the lowest level at metamorphic climax in M. nipponense. We speculate that the expression patterns of Mnsxll and Mnsxl2 during larval development are necessary to stimulate pre-metamorphic larval changes of morphology and initiate metamorphosis, which is consistent with previous studies showing that the sex differentiation and sexually dimorphic development of the crustaceans begin after metamorphosis (Lee et al., 1994; Zhao, 2009). As is already known, changes of external sexual forms occur before those of the internal sexual forms (Zhu et al., 2011). In a follow-up study (unpublished), our laboratory found from histological sections that primordial germ cells (PGCs) of M. nipponense appeared in 10 15 days of post-larvae, and its external sexual forms occurred at 5 10 days post-larvae. The time of Mnsxl action in post-larvae tended to correlate with the occurrence time of PGCs and external sexual forms of M. nipponense, suggesting that the Mnsxl homologs were involved in sex differentiation and external sexual forms in M. nipponense.

In conclusion, our results together with literature evidence suggest that the Mnsxl homologs play important roles in the embryonic development and sex differentiation of $M$. nipponense. This study advances our understanding of the multiple biological functions of the Mnsxl genes, and lays a foundation for researching the regulation mechanism of the sexdetermination pathway in M. nipponense.

\section{ACKNOWLEDGMENTS}

Research supported by the National Natural Science Foundation of China (\#31272654), the National "Twelfth Five-Year" Plan for Science \& Technology Support (\#2012BAD26B04; \#2012BAD25B07), the National Natural Science Foundation of Jiangsu Province (BK\#2012091), The Science \& Technology Supporting Program of Jiangsu Province (BE\#2012334), and the Freshwater Fisheries Research Center, China Central Governmental Research Institutional Basic Special Research Project from the Public Welfare Fund (\#2013JBFT07). 


\section{REFERENCES}

Bell LR, Maine EM, Schedl P and Cline TW (1988). Sex-lethal, a Drosophila sex determination switch gene, exhibits sexspecific RNA splicing and sequence similarity to RNA binding proteins. Cell 55: 1037-1046.

Bopp D, Bell LR, Cline TW and Schedl P (1991). Developmental distribution of female-specific Sex-lethal proteins in Drosophila melanogaster. Genes Dev. 5: 403-415.

Budd GE and Telford MJ (2009). The origin and evolution of arthropods. Nature 457: 812-817.

Bureau of Fishery, Ministry of Agriculture, P.R.C. (2009). Fisheries Economic Statistics. China Agricultural Press, Beijing.

Casper AL and Van Doren M (2009). The establishment of sexual identity in the Drosophila germline. Development 136: 3821-3830.

ChenY, Zhu Q, Chen H, Zhu XL, et al. (2012). The morphological and histological observation of embryonic development in the oriental river prawn Macrobrachium nipponense. J. Shanghai Ocean Univ. 21: 33-40.

Cline TW (1978). Two closely linked mutations in Drosophila melanogaster that are lethal to opposite sexes and interact with daughterless. Genetics 90: 683-698.

Cline TW and Meyer BJ (1996). Vive la difference: males vs females in flies vs worms. Annu. Rev. Genet. 30: 637-702.

Cline TW, Dorsett M, Sun S, Harrison MM, et al. (2010). Evolution of the Drosophila feminizing switch gene sex-lethal. Genetics 186: 1321-1336.

Glenner H, Thomsen PF, Hebsgaard MB, Sorensen MV, et al. (2006). Evolution. The origin of insects. Science 314: 1883-1884.

Jin ZX, Ye HH, Li SJ, Huang HY, et al. (2003). Role of nervous organs in stimulating ovarian maturation in the mud crab Scylla serrata: an in vitro study. Mar. Sci. 27: 72-74.

Kato Y, Kobayashi K, Watanabe H and Iguchi T (2011). Environmental sex determination in the branchiopod crustacean Daphnia magna: deep conservation of a Doublesex gene in the sex-determining pathway. PLoS Genet. 7: e1001345.

Keyes LN, Cline TW and Schedl P (1992). The primary sex determination signal of Drosophila acts at the level of transcription. Cell 68: 933-943.

Kulkarni GK, Glade L and Fingerman M (1991). Oogensis and effects of neuroendocrine tissues on in vitro synthesis of protein by the ovary of the red swamp crayfish, Procambarus clarkii (Girard). J. Crustacean Biol. 11: 513-522.

Lagos D, Ruiz MF, Sanchez L and Komitopoulou K (2005). Isolation and characterization of the Bactrocera oleae genes orthologous to the sex determining Sex-lethal and doublesex genes of Drosophila melanogaster. Gene 348: 111-121.

Lee T-H, Yamauchi M and Yamazaki F (1994). Sex differentiation in the crab Eriocheir japonicas (Decapoda, Grapsidae). Invert. Reprod. Dev. 25: 123-138.

Li Y, Qian YQ, Ma WM and Yang WJ (2009). Inhibition mechanism and the effects of structure on activity of male reproduction-related peptidase inhibitor Kazal-type (MRPINK) of Macrobrachium rosenbergii. Mar. Biotechnol. 11: $252-259$

Livak KJ and Schmittgen TD (2001). Analysis of relative gene expression data using real-time quantitative PCR and the $2^{-\Delta \Delta \mathrm{C}_{\mathrm{T}}}$ method. Methods 25: 402-408.

Maine EM, Salz HK, Schedl P and Cline TW (1985). Sex-lethal, a link between sex determination and sexual differentiation in Drosophila melanogaster. Cold Spring Harb. Symp. Quant. Biol. 50: 595-604.

Meeratana P and Sobhon P (2007). Classification of differentiating oocytes during ovarian cycle in the giant freshwater prawn, Macrobrachium rosenbergii de man. Aquaculture 270: 259-258.

Müller-Holtkamp F (1995). The Sex-lethal gene homologue in Chrysomya rufifacies is highly conserved in sequence and exon-intron organization. J. Mol. Evol. 41: 467-477.

Penalva LO and Sanchez L (2003). RNA binding protein sex-lethal (Sxl) and control of Drosophila sex determination and dosage compensation. Microbiol. Mol. Biol. Rev. 67: 343-59, table.

Qiao H, Fu H, Jin S, Wu Y, et al. (2012). Constructing and random sequencing analysis of normalized cDNA library of testis tissue from oriental river prawn (Macrobrachium nipponense). Comp. Biochem. Physiol. Part D Genom. Proteomics 7: 268-276.

Qiu GF, Yamano K and Unuma T (2005). Cathepsin C transcripts are differentially expressed in the final stages of oocyte maturation in kuruma prawn Marsupenaeus japonicus. Comp. Biochem. Physiol. B Biochem. Mol. Biol. 140: 171-181.

Ruiz MF, Goday C, Gonzalez P and Sanchez L (2003). Molecular analysis and developmental expression of the Sex-lethal gene of Sciara ocellaris (Diptera order, Nematocera suborder). Gene. Expr. Patterns 3: 341-346.

Saccone G, Peluso I, Artiaco D, Giordano E, et al. (1998). The Ceratitis capitata homologue of the Drosophila sexdetermining gene sex-lethal is structurally conserved, but not sex-specifically regulated. Development 125: 14951500 . 
Salz HK, Maine EM, Keyes LN, Samuels ME, et al. (1989). The Drosophila female-specific sex-determination gene, Sexlethal, has stage-, tissue-, and sex-specific RNAs suggesting multiple modes of regulation. Genes Dev. 3: 708-719.

Schütt C and Nöthiger R (2000). Structure, function and evolution of sex-determining systems in Dipteran insects. Development 127: 667-677.

Serna E, Gorab E, Ruiz MF, Goday C, et al. (2004). The gene Sex-lethal of the Sciaridae family (order Diptera, suborder Nematocera) and its phylogeny in dipteran insects. Genetics 168: 907-921.

Sievert V, Kuhn S, Paululat A and Traut W (2000). Sequence conservation and expression of the sex-lethal homologue in the fly Megaselia scalaris. Genome 43: 382-390.

Siwicki KK and Kravitz EA (2009). Fruitless, doublesex and the genetics of social behavior in Drosophila melanogaster. Curr. Opin. Neurobiol. 19: 200-206.

Traut W, Niimi T, Ikeo K and Sahara K (2006). Phylogeny of the sex-determining gene Sex-lethal in insects. Genome 49: 254-262.

Ventura T, Manor R, Aflalo ED, Weil S, et al. (2012). Timing sexual differentiation: full functional sex reversal achieved through silencing of a single insulin-like gene in the prawn, Macrobrachium rosenbergii. Biol. Reprod. 86: 90.

Vied C and Horabin JI (2001). The sex determination master switch, Sex-lethal, responds to Hedgehog signaling in the Drosophila germline. Development 128: 2649-2660.

Vied C, Halachmi N, Salzberg A and Horabin JI (2003). Antizyme is a target of sex-lethal in the Drosophila germline and appears to act downstream of hedgehog to regulate sex-lethal and cyclin B. Dev. Biol. 253: 214-229.

Wilkins AS (1995). Moving up the hierarchy: a hypothesis on the evolution of a genetic sex determination pathway. BioEssays 17: 71-77.

Zhang F, Chen L, Wu P, Zhao W, et al. (2010). cDNA cloning and expression of Ubc9 in the developing embryo and ovary of Oriental river prawn, Macrobrachium nipponense. Comp. Biochem. Physiol. B Biochem. Mol. Biol. 155: 288-293.

Zhao GF, Li GL and Zhu CH (2009). Preliminary study on sex differentiation of Litopenaeus vannamei. J. Guangdong Ocean Univ. 29: 19-23.

Zhu CH, Xue HB, Li YJ, Huang GZ, et al. (2011). Effcets of 4 nonylphenol on growth and sex differentiation in Macrobrachium rosenbergii. J. Fish. China 33: 365-371. 